\author{
A. S. Merenga \\ C. M. Papadakis \\ F. Kremer \\ J. Liu \\ A. F. Yee
}

\section{Broadband dielectric spectroscopy of the inter- and intramolecular dynamics of a series of random polyester copolymers}

Received: 28 September 2000

Accepted: 29 January 2001

\section{A. S. Merenga $\cdot$ C. M. Papadakis \\ F. Kremer $(\bowtie)$ \\ Faculty of Physics and Earthsciences \\ University of Leipzig, Linnéstrasse 5 \\ D-04103 Leipzig, Germany \\ e-mail: kremer@physik.uni-leipzig.de \\ Fax: + 49-341-9732599}

J. Liu · A. F. Yee

Macromolecular Science and Engineering

University of Michigan, Ann Arbor

Michigan, 48109-2136, USA

Present address: A. S. Merenga

Department of Physics

Kenyatta University, Box 43844

Nairobi, Kenya

\begin{abstract}
Broadband dielectric spectroscopy $\left(1-10^{6} \mathrm{~Hz}, 183-423 \mathrm{~K}\right)$ and differential scanning calorimetry are employed to analyze the inter- and intramolecular dynamics of a series of random copolymers based on poly(ethylene terephthalate) and poly(1,4-cyclohexylene dimethylene terephthalate). In addition to an interfacial relaxation ( $\alpha^{*}$-process), three dielectric relaxation processes are observed: The $\alpha$-relaxation ("dynamic glass transition") and two secondary relaxations $(\beta$ - and $\beta^{*}$-relaxations). The $\alpha$-relaxation depends sensitively on the composition of the copolymer and shows a rapid slowing down with increasing
\end{abstract}

content of cyclohexylene dimethylene (CHDM) linkages. Besides the $\beta$-relaxation, attributed to local motion of the ester group, an additional process $\left(\beta^{*}\right.$-relaxation) is observed on introducing the CHDM linkages. Increasing the content of the latter reduces the strength of the $\beta$-relaxation strongly and increases its activation energy by more than $30 \%$. This proves that owing to interactions between the cylohexylene rings and the ester group the $\beta$-relaxation no longer has local character only.

Key words Polymer dynamics · Random copolymers - Broadband dielectric spectroscopy

\section{Introduction}

Several studies have been carried out on the secondary relaxation behavior of poly(ethylene terephthalate) (PET) and its plasticized form [1-4]. The motivation in these studies lies in the fact that the secondary relaxation process is related to the mechanical properties of the polymer and in the industrial importance of the effect of plasticization on polymer properties. For instance, blending of poly(alkyl terephthalate)-based polymers has been widely used to modify their properties.

Alternatively, copolymers represent a way to tailor the macroscopic properties of polymers. The copolymer properties may differ markedly from those of the corresponding homopolymers because of specific interactions between the different monomers. Copolymerization allows manipulation of the softening point of the material, with the glass transition usually varying monotonically with composition between those of the two homopolymers. Thus, studies looking into the effect of copolymerizing PET on the molecular dynamics have become of increasing interest for basic and applied research $[5,6]$.

Copolymerization can significantly change the molecular environment of the respective monomers. This may lead to new backbone or side-chain conformational states with a wide distribution of orientational energy barriers. The motion of individual molecules will, therefore, be determined by both inter- and intramolecular interactions within their immediate surroundings. The size of the region in which a molecule is influenced by its neighbors depends on the specific interactions between the copolymer components.

As early as in 1950, Reddish [7] investigated the dielectric losses in poly(1,4-cyclohexylene dimethylene terephthalate) (PCT). He found that the secondary loss peak in PCT is slightly higher in temperature and slightly lower in magnitude compared to that in PET at 
$60 \mathrm{~Hz}$. The activation energy of the secondary relaxation in PCT determined in that study was $55.3 \mathrm{~kJ} / \mathrm{mol}$.

In their dynamic mechanical measurements on a series of copolymers based on PET and PCT, Chen et al. [8] found that the secondary loss peak temperature increases from $-75^{\circ} \mathrm{C}$ for PET to $-70{ }^{\circ} \mathrm{C}$ for PCT. In contrast to the observations made by Reddish, the intensity of the secondary loss peak of PCT was higher than that of PET. The discrepancy between the two studies is due to the fact that the motions involving specific groups monitored in these two techniques are not necessarily the same. Chen et al. [8] attributed this peak shift in temperature to the cooperative motion of the cyclohexylene rings and neighboring terephthalate units. On the basis of additional dipolar rotational spinecho ${ }^{13} \mathrm{C}$ NMR results, it was shown that the motion of the cyclohexylene rings increased with the PCT content, and it was suggested that the rings can undergo chairchair transitions depending on how they are substituted [8]. Details on the possible conformations and transitions of the cyclohexylene rings can be found in Ref. [8].

Recently, Ward et al. [3] carried out mechanical and dielectric measurements on PET/additive blends. They observed that the secondary mechanical peak is composed of a high-temperature component associated with motions of the phenylene rings and of a low-temperature peak associated with motion of the carbonyl groups [3]; however, the secondary dielectric peak could not be resolved into two peaks, but it had the same activation energy as the low-temperature component of the mechanical peak. Based on deuterium NMR experiments, Ward et al. [9] confirmed that the high-temperature component, which does not appear in the dielectric spectra, must be related to motions of nonpolar phenyl ring motion and not the glycol units. They also observed that the presence of low-molecular-weight additives suppresses the high-temperature component of the

Table 1 Mole percentages of poly(ethylene terephthalate) (PET) and poly(1,4-cyclohexylene dimethylene terephthalate) $(P C T)$, repeat units, number-average $\left(\bar{M}_{\mathrm{n}}\right)$ and weight-average $\left(\bar{M}_{\mathrm{w}}\right)$ molar masses determined by gel permeation chromatography as PET mechanical peak, leaving the low-temperature component virtually unaltered [9]. They finally concluded that the extremely small motions in the glycol units do not significantly contribute to the secondary relaxation processes.

In this article, we present broadband dielectric spectroscopy measurements on a series of polyester random copolymers based on PET and PCT both in the glassy and rubbery states with the aim of investigating how the cyclohexylene dimethylene linkages affect the molecular dynamics.

\section{Experimental}

Sample preparation

A series of random copolyesters derived from dimethyl terephthalate and ethylene glycol and/or 1,4-cyclohexylene dimethanol (CHDM) were used in this study. Details of the synthesis are described elsewhere [8]. The copolymers were based on PET with the following increasing mole percentages of CHDM: 3.5, 31, 62, and $81 \mathrm{~mol} \%$. Note that the PCT homopolymer has $100 \mathrm{~mol} \%$ CHDM. In all samples, the cyclohexylene rings in the copolyesters and in PCT had a trans-to-cis molar ratio of $68: 32$. The structures of the repeat units of the polymers as well as their properties are given in Table 1. The materials were in the form of injectionmolded bars. The films for dielectric measurements were prepared by compression molding between two gold-plated brass electrodes $(10 \mathrm{~mm}$ in diameter) separated by $50-\mu \mathrm{m}$-thick glass fibers at the corresponding molding temperatures and by subsequent fast quenching in ice-water to obtain amorphous samples. The glasstransition temperatures $\left(T_{\mathrm{g}}\right)$ were determined using a Series 7 Perkin Elmer differential scanning calorimeter at a heating rate of $10 \mathrm{~K} / \mathrm{min}$. The densities of the samples were determined by using a gradient (chloroform/toluene) column method at $296 \mathrm{~K}$.

\section{Dielectric measurements}

Dielectric measurements in the frequency range $1-10^{6} \mathrm{~Hz}$ were performed using a Solartron-Schlumberger SI 1260 (sample

equivalents, glass-transition temperatures measured by differential scanning calorimetry $(D S C)$ at a heating rate of $10 \mathrm{~K} / \mathrm{min}$, and densities determined by the (chloroform/toluene) density gradient column method

\begin{tabular}{llllll}
\hline PET:PCT ratio & Repeat unit & $\bar{M}_{\mathrm{n}}(\mathrm{g} / \mathrm{mol})$ & $\bar{M}_{\mathrm{w}}(\mathrm{g} / \mathrm{mol})$ & $T_{\mathrm{g}} \mathrm{DSC}(\mathrm{K})$ & $\rho\left(\mathrm{g} / \mathrm{cm}^{3}\right)$ \\
\hline $100: 0($ PET) & $\mathrm{a}$ & 22,000 & 42,500 & 346 & 1.321 \\
$96.5: 3.5$ & Copolymer based on PET and PCT & 25,300 & 49,900 & 352 & 1.290 \\
$69: 31$ & Copolymer based on PET and PCT & 22,700 & 44,900 & 352 & 1.270 \\
$38: 62$ & Copolymer based on PET and PCT & 23,700 & 46,200 & 354 & 1.231 \\
$19: 81$ & Copolymer based on PET and PCT & 22,500 & 41,800 & 361 & 1.211 \\
$0: 100$ (PCT) & b & 15,500 & 30,500 & 369 & 1.205 \\
\hline
\end{tabular}

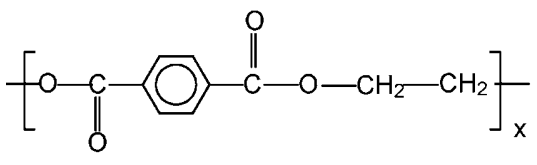

(a)

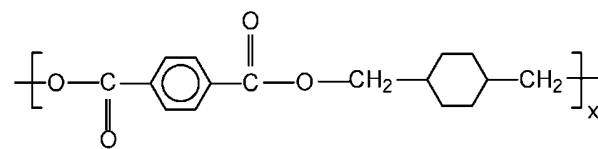

(b) 
diameter $10 \mathrm{~mm}$ ) frequency response analyzer accompanied by a Novocontrol buffer amplifier (broadband dielectric converter). The measurements covered a temperature range from 183 to $423 \mathrm{~K}$. The sample temperature was controlled by using a nitrogen gas controlled heating system (Novocontrol Quatro) having a stability of $\pm 0.5 \mathrm{~K}$. To perform isothermal crystallization measurements, a newly quenched sample was brought to the crystallization temperature $\left(T=T_{\mathrm{g}}+38 \mathrm{~K}\right)$ at a constant heating rate of $20 \mathrm{~K} / \mathrm{min}$. After reaching this temperature, the dielectric measurements were performed in the $10-10^{6} \mathrm{~Hz}$ frequency range as a function of crystallization time. Each frequency scan required about 2 min. The experimental conditions were carefully chosen both to avoid significant precrystallization of the sample during the heating process and to place the dynamic $\alpha$-relaxation process in the middle of our experimental frequency domain window.

In order to describe the dielectric spectra quantitatively, a superposition of model functions according to Havriliak and Negami [10] and a conductivity contribution were fitted (Novocontrol Winfit) to the isothermal dielectric loss data, $\varepsilon^{\prime \prime}$ :

$\varepsilon^{\prime \prime}=\frac{\sigma_{0}}{\varepsilon_{0}} \frac{a}{\omega^{s}}-\sum_{k=1}^{3} \operatorname{Im}\left[\frac{\Delta \varepsilon_{k}}{\left[1+\left(i \omega \tau_{k}\right)^{\alpha_{k}}\right]^{\beta_{k}}}\right]$.

In this notation, $\varepsilon_{o}$ is the vacuum permittivity, $\sigma_{\mathrm{o}}$ the direct current conductivity, $\Delta \varepsilon$ the dielectric strength and $\tau$ the mean relaxation time. The index $k$ refers to the different processes which contribute to the dielectric response. $\alpha_{\mathrm{k}}$ and $\beta_{\mathrm{k}}$ describe the symmetric and asymmetric broadening of the relaxation time distribution. The first term on the right-hand side of Eq. (1) is caused by translational motion of mobile charge carriers. For Ohmic behavior, $s$ equals unity, deviations $(s<1)$ are caused by electrode polarization. $a$ is a factor having the dimensionality $[a]=\mathrm{Hz}^{1-\mathrm{s}}$.

\section{Results and discussion}

\section{$\alpha$-relaxation}

A plot of the dielectric loss, $\varepsilon^{\prime \prime}$, versus frequency in the homopolymers and in two copolymers at $383 \mathrm{~K}$ is shown in Fig. 1. Starting with PET, two peaks are observed. The higher-frequency ( $\alpha$-process) and lowerfrequency $\left(\alpha^{*}\right.$-process or interfacial relaxation) processes are related to the relaxation of dipoles in the free amorphous and the intercrystalline domains, respectively [11]. By comparison of the dielectric absorption in PET with that of the copolymer and of PCT two features are evident: firstly, the copolymers also exhibit two peaks, whereas only one peak is observed in PCT; secondly, the $\alpha$-peak shift shows a monotonous dependence on the CHDM content, whereas the $\alpha^{*}$-peak shift does not.

The temperature dependence of the relaxation time of the $\alpha$-process can be described by the Williams-LandelFerry (WLF) equation [12] (Fig. 2), which is given by

$\log \left(\frac{\tau(T)}{\tau\left(T_{\mathrm{g}}^{B D S}\right)}\right)=\frac{C_{1}\left(T-T_{\mathrm{g}}^{\mathrm{BDS}}\right)}{C_{2}+T-T_{\mathrm{g}}^{\mathrm{BDS}}}$.

with $C_{1}$ and $C_{2}$ being constants and $T_{\mathrm{g}}{ }^{\mathrm{BDS}}$ the glasstransition temperature found using broadband dielectric

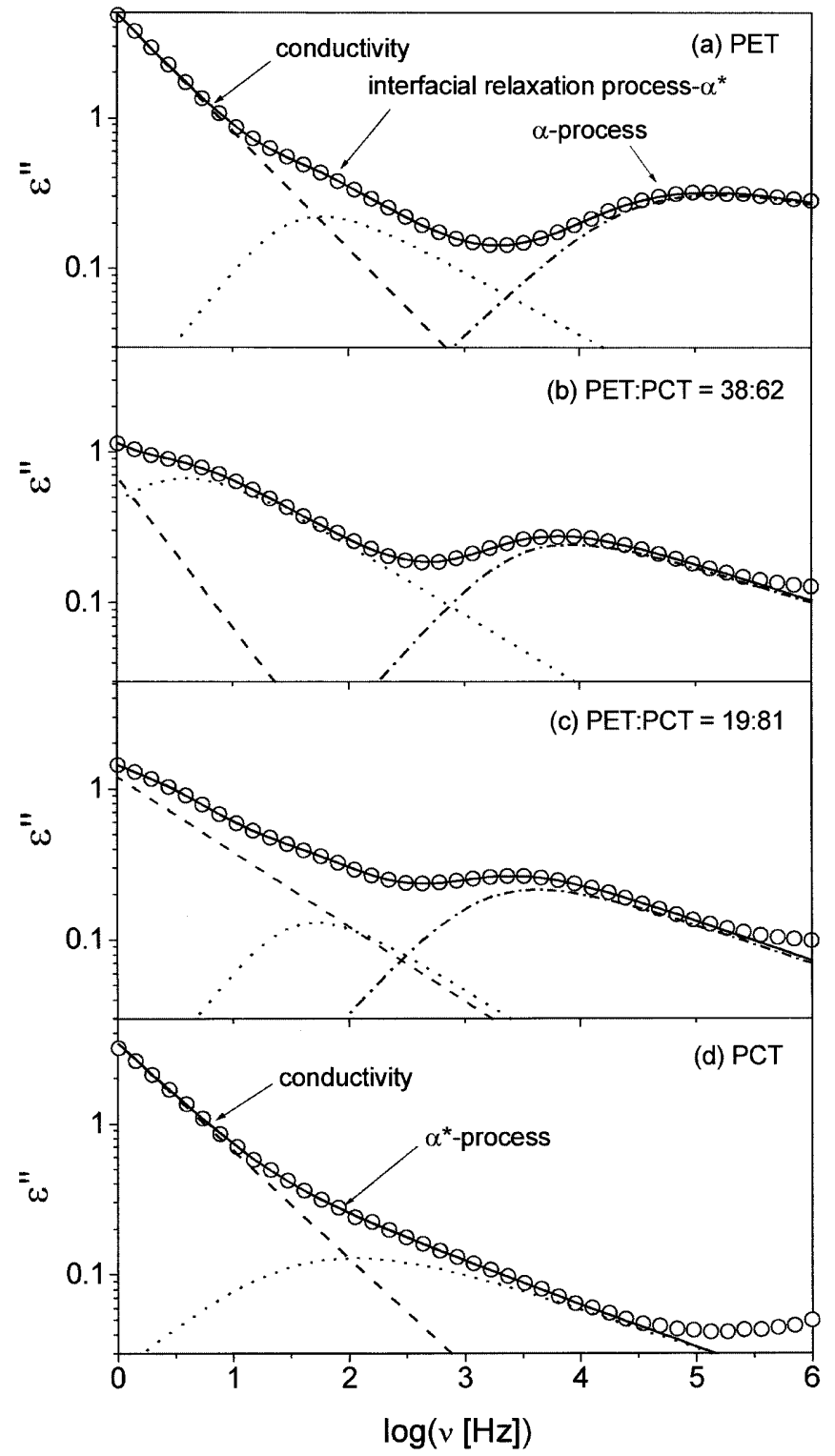

Fig. 1 Double-logarithmic representation of the dielectric loss, $\varepsilon^{\prime \prime}$, as a function of frequency, $v$, for the $\alpha$ - and $\alpha^{*}$-processes at $383 \mathrm{~K}$ in a poly(ethylene terephthalate) $(P E T)$, in copolymers having PET: poly(1,4-cyclohexylene dimethylene terephthalate) $(P C T)$ ratios of b 38:62 and c 19:81, and in d PCT. The data are described as a superposition (solid lines) of the relaxation process (according to Havriliak-Negami, Eq. 1, dotted and dash-dotted lines) and a conductivity contribution (dashed lines). The increase in $\varepsilon^{\prime \prime}$ at high frequencies arises from contributions from the low-frequency tail of the $\beta$ - and $\beta^{*}$-processes

spectroscopy. A summary of the WLF parameters obtained for all the samples investigated is given in Table 2 . The $T_{\mathrm{g}}$ values as measured calorimetrically and dielectrically do not coincide within the limits of experimental accuracy. This is caused by the fact that, in contrast to calorimetry, the dielectric method senses the fluctuations of the dipolar units only. 
Fig. 2 Temperature dependence of the relaxation rates of the $\alpha$-process (solid symbols) and the $\alpha^{*}$-process (open symbols) for various PET-PCT copolymers. The symbols correspond to copolymers with PET:PCT ratios of 100:0 (squares), 96.5:3.5 (circles), 69:31 (upper triangles), 38:62 (lower triangles), 19:81 (diamonds), and 0:100 (stars)

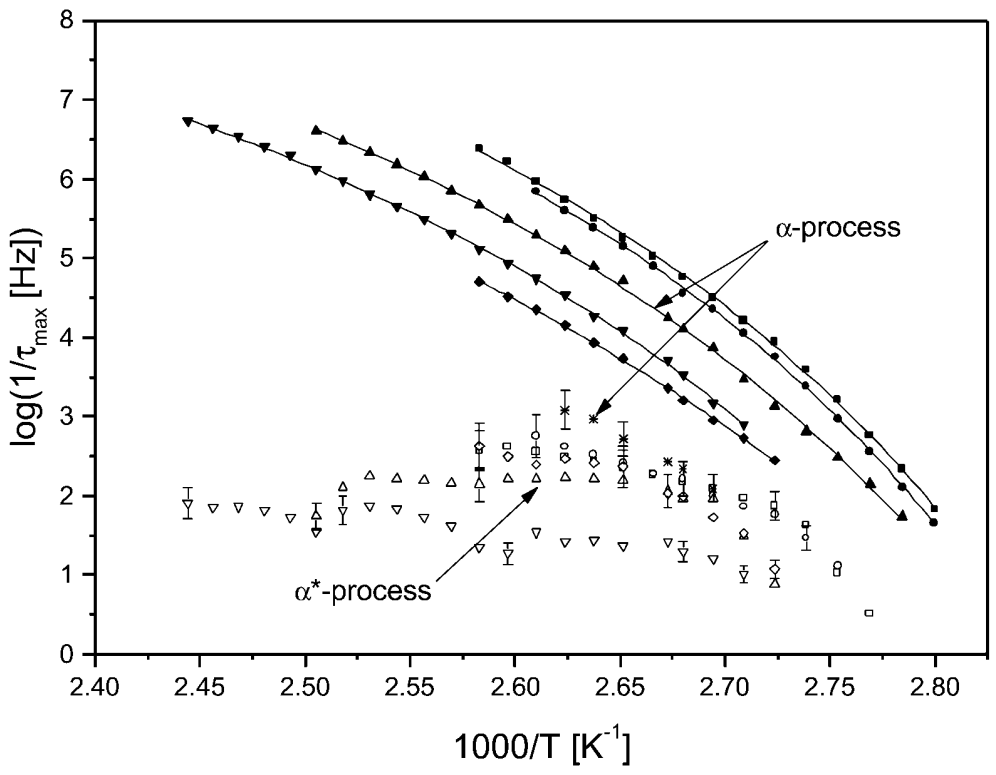

Table 2 Williams-Landel-Ferry fit parameters of the $\alpha$-relaxation of PET-PCT copolymers together with the glass-transition temperatures from DSC

\begin{tabular}{lllll}
\hline $\begin{array}{l}\text { PET:PCT } \\
\text { ratio }\end{array}$ & $\begin{array}{l}C_{1} \\
\pm 2(\mathrm{~K})\end{array}$ & $\begin{array}{l}C_{2} \\
\pm 3(\mathrm{~K})\end{array}$ & $\begin{array}{l}T_{\mathrm{g}}^{\mathrm{BDS}} \\
\pm 2(\mathrm{~K})\end{array}$ & $\begin{array}{l}T_{\mathrm{g}} \text { DSC } \\
\pm 2(\mathrm{~K})\end{array}$ \\
\hline $100: 0$ & 24 & 43 & 358 & 346 \\
$96.5: 3.5$ & 26 & 47 & 359 & 352 \\
$69: 31$ & 25 & 52 & 351 & 352 \\
$38: 62$ & 24 & 54 & 364 & 354 \\
$19: 81$ & 31 & 52 & 367 & 361 \\
\hline
\end{tabular}

A plot of the dielectric loss versus the logarithm of frequency in the homopolymers and two copolymers during isothermal measurements at $T=T_{\mathrm{g}}{ }^{\mathrm{DSC}}+38 \mathrm{~K}$ is shown in Fig. 3. Note the $\alpha^{*}$-process is not present in these spectra (cf. Fig. 1), which may be due to a difference in the thermal history. Figure 4 shows the variation of the relaxation rate of the $\alpha$-process, corresponding to Fig. 3, as a function of crystallization time $\left(t_{\mathrm{c}}\right)$. It can be seen that with time, the relaxation rate of the $\alpha$-process decreases owing to crystal growth. This is observed for lower and higher amounts of CHDM; however, at 31 and $62 \%$ CHDM content, the relaxation rate is virtually unaffected by crystal growth. This behavior is also reflected in our differential scanning calorimetry (DSC) measurements (Fig. 5), where the position of the exotherm (owing to crystallization) above the glass-transition temperature is shifted to higher temperatures with increasing amounts of CHDM. At 31 and $62 \%$ CHDM content the exotherm vanishes and above $62 \%$ its position is shifted to lower temperatures. The observed invariance of the relaxation rate at 31 and $62 \%$ CHDM content during isothermal treatment can be explained intuitively by considering the morphology of this type of copolymer. Typically, homopolymers based on PET or PCT can build up chain-folded lamellae of the crystallizable segments embedded in an amorphous phase. On a larger scale, crystalline lamellae arrange themselves in spherulitic structures. Because of the irregular structure of the copolymers, the relation of the crystallized material to the overall spherulite volume for copolymers is expected to be smaller than for typical spherulites of homopolymers (PET or PCT). The amorphous phase is expected to be less constrained by the crystalline lamellae than when they arrange within more compact spherulitic structures such as those formed by the homopolymer. Additional optical microscopy and combined ${ }^{1} \mathrm{H} /{ }^{13} \mathrm{C}$ NMR measurements [13] which monitor the nucleation density during crystallization could explain this point. Note that with the sample with a PET:PCT ratio of 38:62 the $\alpha^{*}$-process is observed in the dielectric spectra (Fig. 1) even though the DSC scan (Fig. 5) does not show a crystallization exotherm. This may be caused by the fact that the time required for dielectric spectra is much longer than for the DSC scans and that the sample geometry differs in the two methods. Also, in PCT, the $\alpha$-process is absent in the dielectric spectra (Fig. 1), which may be related to the fact that the crystallization exotherm is long and sharp (Fig. 5), i.e. a large part of the sample is crystallized.

As shown in Table 1, the specific volume of the PETPCT copolymers increases with the CHDM content, which may be due to the bulkiness of the cyclohexylene linkages [14-16]. Increasing the free volume usually lowers the temperature of the exothermic peak as 


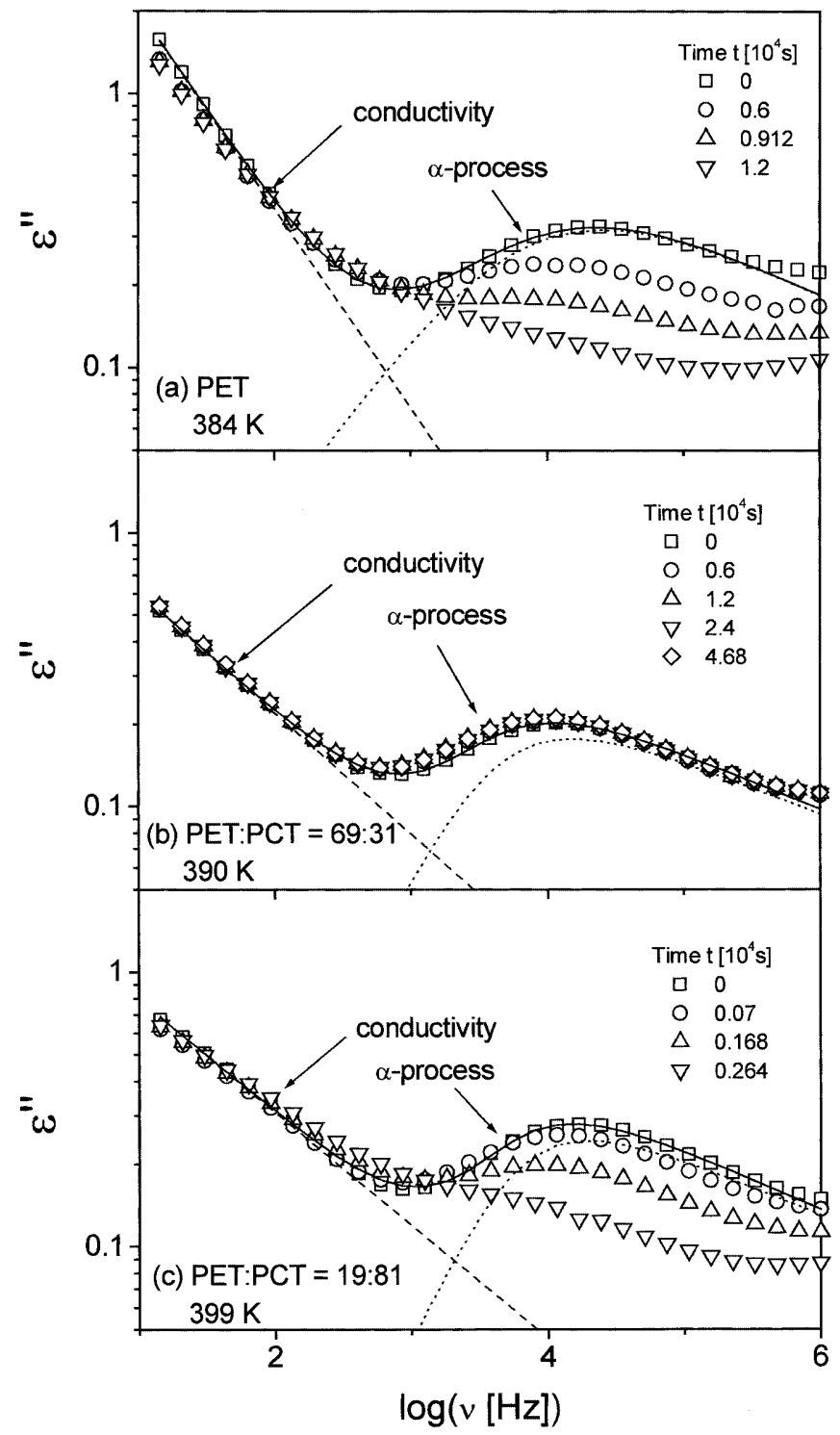

Fig. 3 Double-logarithmic representation of the real-time evolution of $\varepsilon^{\prime \prime}$ as a function of frequency for the $\alpha$-process in a PET and in copolymers having PET:PCT ratios of b 69:31 and $\mathbf{c}$ 19:81 during an isothermal crystallization process at $T=T_{\mathrm{g}}{ }^{\mathrm{DSC}}+38 \mathrm{~K}$. The data are described as a superposition (solid lines) of the relaxation processes (according to Havriliak-Negami, Eq. 1, dotted lines) and conductivity contributions (dashed lines). The increase in $\varepsilon^{\prime \prime}$ at high frequencies arises from contributions from the low-frequency tails of the $\beta$ - and the $\beta^{*}$-processes

suggested by free-volume theories [17-19]. Since our data contrast this notion, another explanation ought to be given.

It can be seen from Fig. 6a that the dielectric strength of the $\alpha$-process decreases with increasing numbers of CHDM linkages, whereas that of the $\alpha^{*}$-process (Fig. 6b) is independent of the CHDM content. It is obvious from a structural point of view that the main dipoles (the carbonyl groups) in homopolymers and copolymers have similar values. With increasing CHDM content, the number density of dipoles decreases by a factor of about 1.6 (going from PET to PCT), as determined from the monomer molar mass and the mass density (Table 1). The height of the $\alpha$-peak, however, decreases by a factor of about 2.7 (Table 3). The decrease in the number density alone thus cannot explain this decrease, and we conclude that the response of the dipoles is affected by constraints induced by crystallization. As in PET, both trans-PCT and cis-PCT homopolymers are known to crystallize when heated above their glass transition [20]. Note also that the introduction of the cyclohexylene rings leads to partial replacement of the more flexible ethylene chains with methylene ones which have less conformational reorientations [21]. This introduces intramolecular constraints which enhance intermolecular coupling. In addition, the presence of bulky cyclohexylene units also reduces the chain mobility [8]. This may reduce the net dipole moment as revealed by the decrease in the dielectric strength (Table 3).

\section{$\beta^{*}$-relaxation}

Plots of the dielectric loss versus the logarithm of the frequency at 216, 258, and $303 \mathrm{~K}$ for PET, a PET-PCT copolymer with ratio 38:62, and for PCT are shown in Fig. 7. In the copolymer and in PCT, a weaker process, named $\beta^{*}$, on the lower-frequency side of the broader process is observed. The fact that this process is absent in PET suggests that this relaxation is due to additional conformational changes induced by the "chair-to-chair" interconversion of the cyclohexylene rings on the adjacent carbonyl group through $\mathrm{C}$ (cyclohexyl)$\mathrm{C}$ (methyl)-O(ether) bonds as illustrated in Fig. 8. This conclusion is supported by recent ${ }^{13} \mathrm{C}$ NMR measurements on PCT which show that the spin-lattice relaxation times in the rotating frame for all carbons in the cyclohexylene ring and the adjacent methylene carbon adjacent to it decrease at the same rate [8]. The temperature dependence of the relaxation time for the $\beta^{*}$-process follows an Arrhenius law (Fig. 9) given by

$\tau=\tau_{\mathrm{o}} \exp \left(E_{\mathrm{a}} / R T\right)$,

where $E_{\mathrm{a}}$ is the activation energy, $R$ is the universal gas constant, and $\tau_{\mathrm{o}}$ is the characteristic time constant related to the attempt frequency in jumping over the energy barrier $E_{\mathrm{a}}$. It holds only for local relaxation processes, which show typical values for $\tau_{\mathrm{o}}$ of about $10^{-13} \mathrm{~s}$. The values of the activation energies (Table 4) are similar to those obtained from mechanical and dielectric measurements performed by Heijboer [16] on acrylic polymers containing cyclohexylene rings pendant to the polymer backbone. 
Fig. 4 Variation of the relaxation rate of the $\alpha$-process as a function of the crystallization time for various PET-PCT copolymers at $T=T_{\mathrm{g}}{ }^{\mathrm{DSC}}+$ $38 \mathrm{~K}$. The symbols correspond to copolymers with PET:PCT ratios of 100:0 $(\square), 96.5: 3.5(\bigcirc)$, 69:31 $(\triangle), 38: 62(\nabla)$, and 19:81 $(\diamond)$

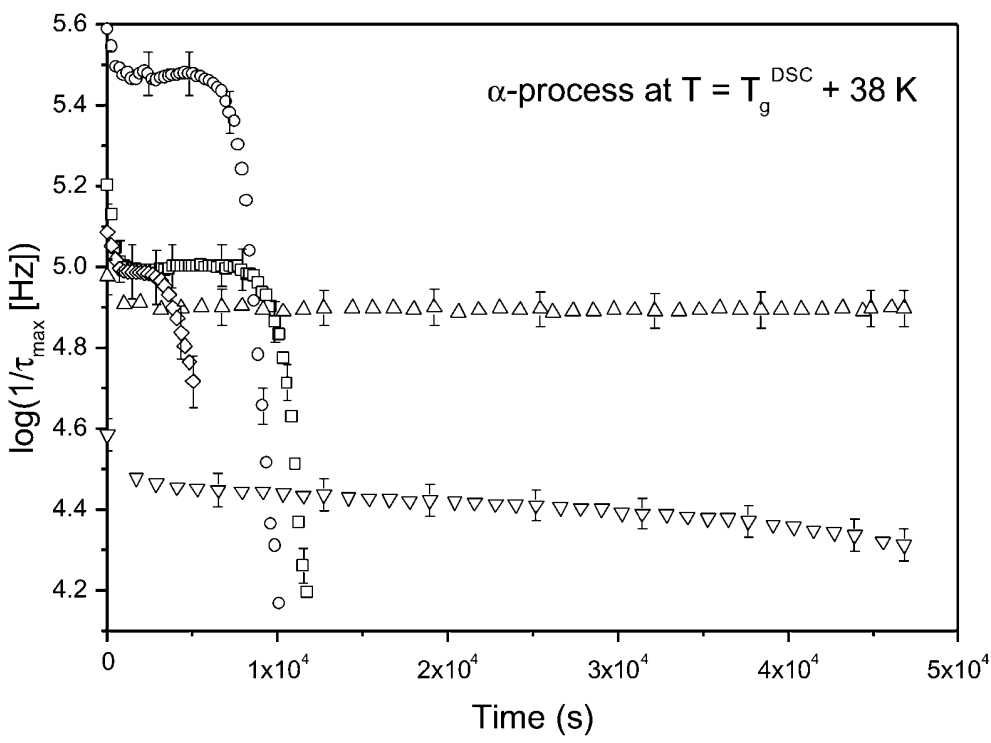

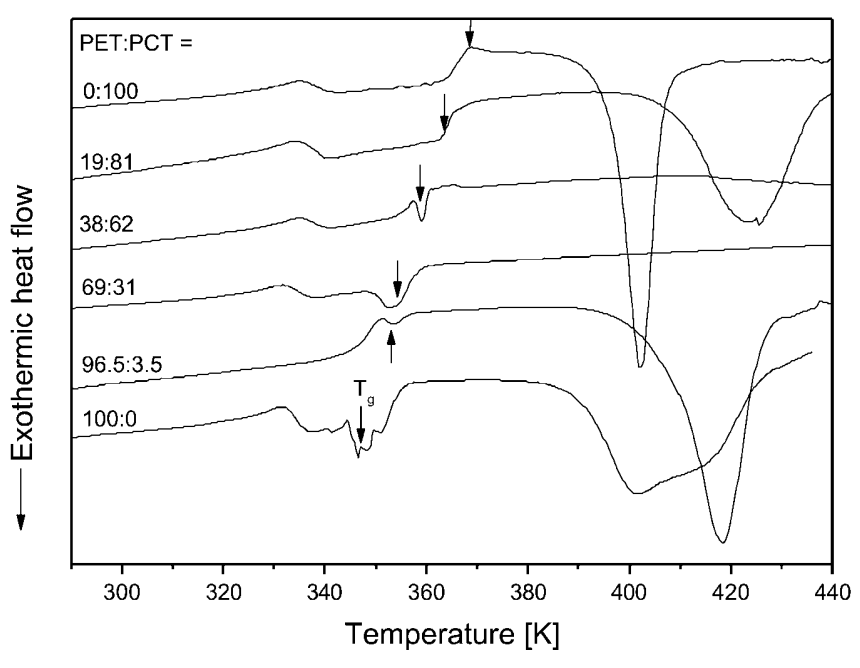

Fig. 5 Differential scanning calorimetry thermograms for the PET, PCT and PET-PCT copolymers investigated. Heating rate $10 \mathrm{~K} / \mathrm{min}$. The arrows indicate the glass-transition temperatures, $T_{\mathrm{g}}$

The process becomes slightly slower with increasing number of CHDM linkages (Fig. 9) and the activation energy increases slightly (Table 4). These observations indicate that the local rearrangement of the cyclohexylene units is more and more hindered by neighboring segments. The intensity of the $\beta^{*}$-process increases with the number of CHDM linkages (Table 3), which justifies the assignment. Dipolar rotational spin-echo ${ }^{13} \mathrm{C}$ NMR on these copolymers confirmed large-amplitude motions of the cyclohexylene ring faster than $10 \mathrm{kHz}$ at $300 \mathrm{~K}$, which compares well to our results (Fig. 9) [8]. Because the cyclohexylene ring is bulky, it is expected that the relaxation process resulting from this unit is slow and weak in dielectric strength, which is indeed the case (Fig. 8, Table 3).

\section{$\beta$-relaxation}

The broader and stronger process $(\beta)$ in Fig. 7 is observed in all the samples. It is assigned to the wellknown librational motions of the carbonyl groups [1, 3 , 5]. The assignment is justified by the fact that its activation energy (determined in this study to be $61 \mathrm{~kJ} /$ mol for PET) compares well with that of librational motions of the carbonyl groups in other systems, such as side-chain liquid crystals [22]. The $\beta$-relaxation may couple to the $180^{\circ}$ flips of the phenyl groups as shown by comparative dielectric and NMR experiments [22]. With increasing numbers of CHDM linkages, the activation energy of the $\beta$-relaxation increases dramatically (Table 4, Fig. 9). It obeys a type of "compensation rule" $[23,24]$ (Meyer-Neldel rule) exhibiting a correlation between the activation energy, $E_{\mathrm{a} \beta}$, and the characteristic relaxation rate in the high-temperature limit, $v_{o}$ (Fig. 10). The compensation behavior in polymeric materials is often considered as indicative of cooperative molecular movements $[25,26]$ and has been ascribed to the relaxation of entities with variable length [27]. The distribution of the activation energy displays, in this context, motions which are hierarchically correlated with a disperse spectrum of movements. Schönhals and coworkers [28, 29] have shown that side-chain liquid-crystalline polymers show similarly high characteristic relaxation rates, which they attributed to cooperative reconfiguration processes within the mesophase. In our case, the presence of 

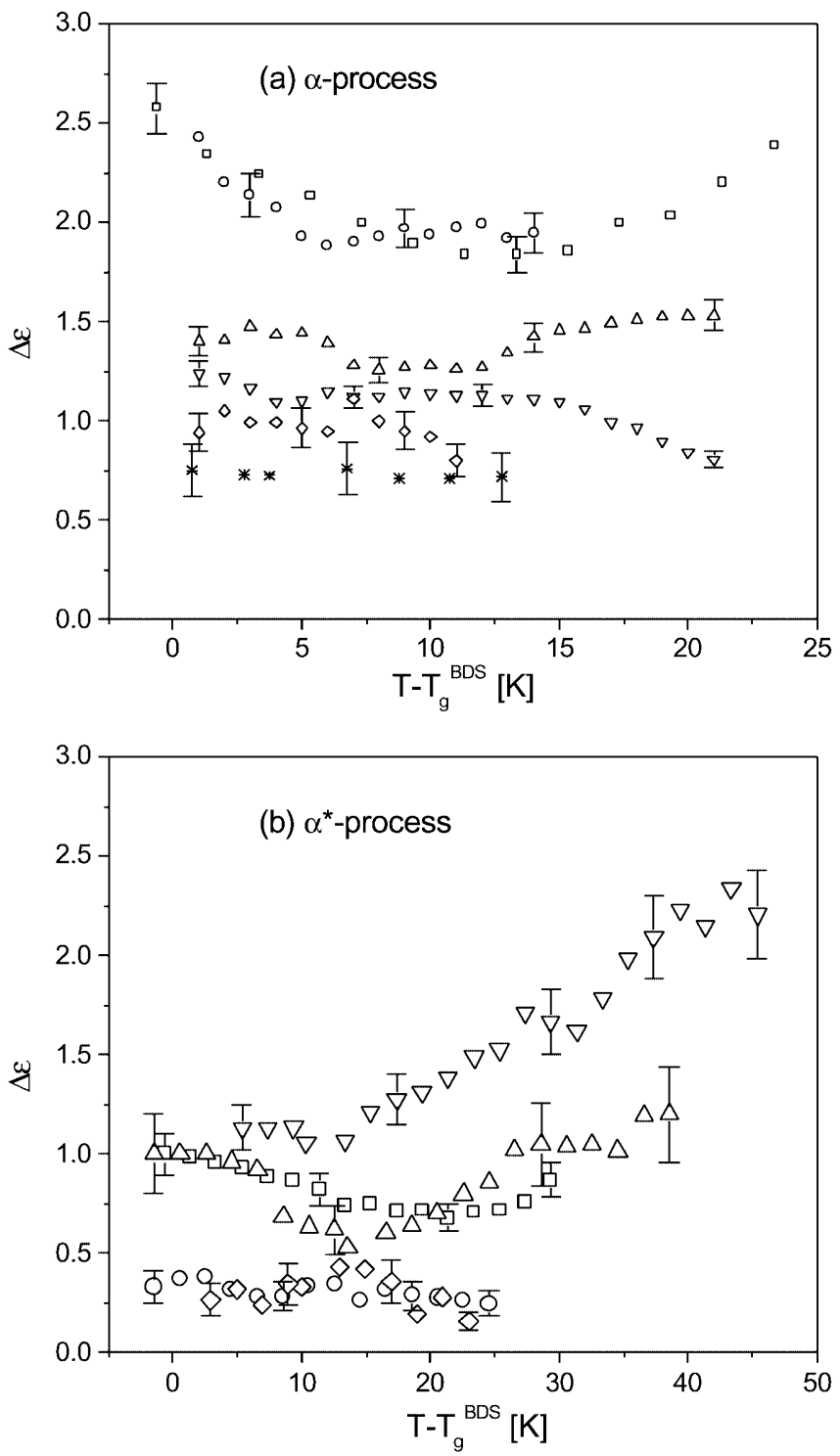

Fig. 6 Relaxation strengths, $\Delta \varepsilon$, of a the $\alpha$-process and $\mathbf{b}$ the $\alpha^{*}$ process as a function of $T-T_{\mathrm{g}} \mathrm{BDS}$. The symbols correspond to copolymers with PET:PCT ratios of 100:0 $(\square), 96.5: 3.5(\bigcirc), 69: 31(\Delta)$, 38:62 $(\nabla), 19: 81(\diamond)$, and 0:100 $\left(^{*}\right)$

Table 3. Dielectric strengths, $\Delta \varepsilon$, of the $\alpha-, \beta^{*}$-, and $\beta$-relaxations

\begin{tabular}{|c|c|c|c|}
\hline $\begin{array}{l}\text { PET:PCT } \\
\text { ratio }\end{array}$ & $\begin{array}{l}\alpha \text {-relaxation: } \Delta \varepsilon \text { at } \\
T-T_{\mathrm{g}}^{\mathrm{BDS}}=15 \mathrm{~K}\end{array}$ & $\begin{array}{l}\beta^{*} \text {-relaxation: } \\
\Delta \varepsilon \text { at } 234 \mathrm{~K}\end{array}$ & $\begin{array}{l}\beta \text {-relaxation: } \\
\Delta \varepsilon \text { at } 258 \mathrm{~K}\end{array}$ \\
\hline 100:0 (PET) & 1.89 & - & 0.87 \\
\hline $96.5: 3.5$ & 1.89 & - & 0.87 \\
\hline $69: 31$ & 1.29 & 0.014 & 0.52 \\
\hline $38: 62$ & 1.15 & 0.020 & 0.49 \\
\hline 19:81 & 1.00 & 0.026 & 0.40 \\
\hline 0:100 (РCT) & 0.71 & 0.031 & 0.35 \\
\hline
\end{tabular}

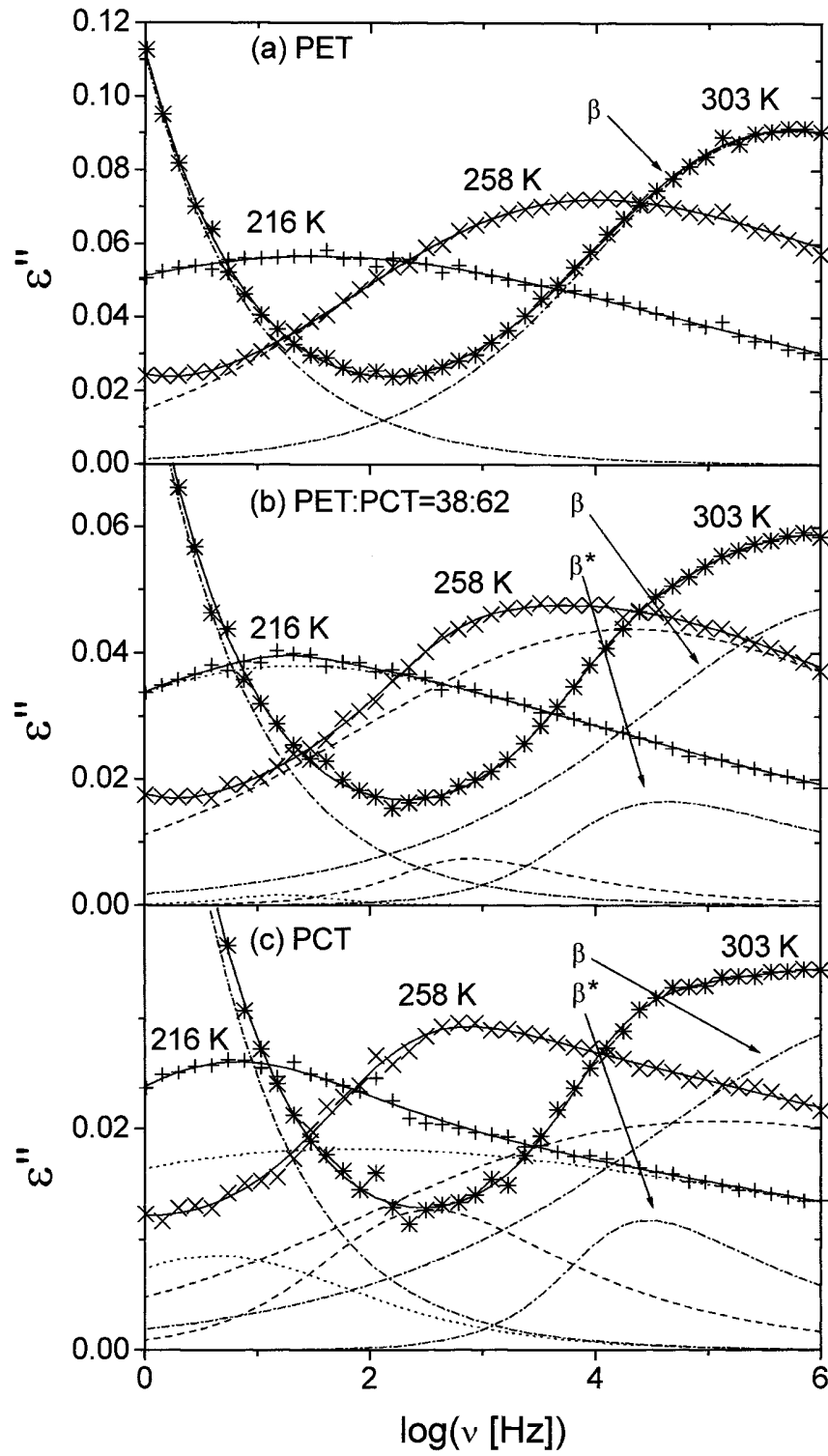

Fig. $7 \varepsilon^{\prime \prime}$ as a function of the logarithm of $v$ for a PET, $\mathbf{b}$ the copolymer having a PET:PCT ratio of 38:62, and c PCT at $216 \mathrm{~K}$ $(+), 258 \mathrm{~K}(\times)$, and $303 \mathrm{~K}\left({ }^{*}\right)$. The data are described as a superposition (solid line) of the relaxation processes (according to Havriliak-Negami, Eq. 1) and a conductivity contribution: $216 \mathrm{~K}$ $(\cdots \cdots \cdots) ; 258$ K (- . . - ) ; $303 \mathrm{~K}(-. \cdots \ldots)$

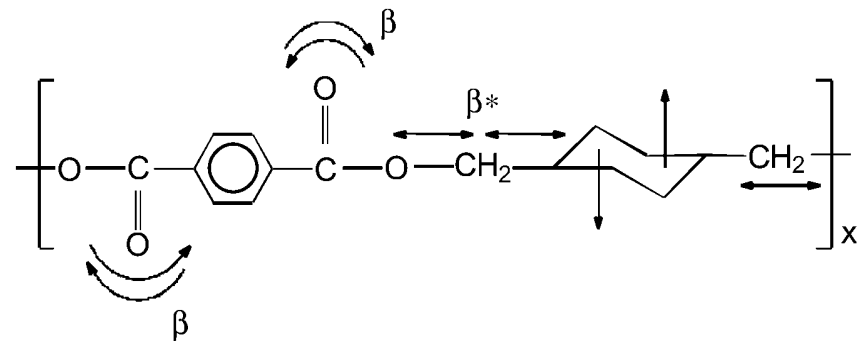

Fig. 8 Schematic illustration of the $\alpha$-, $\beta^{*}$-, and $\beta$-processes in the PET-PCT copolymers investigated 


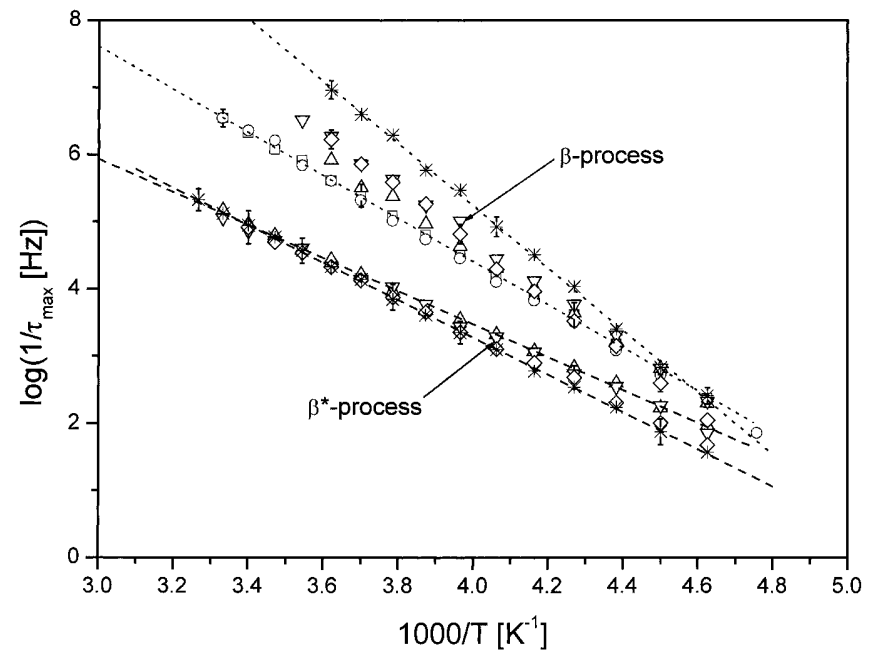

Fig. 9 Temperature dependence of the relaxation rates of the $\beta^{*}$ - and $\beta$-processes for various PET-PCT copolymers. The symbols correspond to PET:PCT ratios of 100:0 $(\square), 96.5: 3.5(\bigcirc), 69: 31(\Delta), 38: 62$ $(\nabla), 19: 81(\diamond)$, and 0:100 $\left(^{*}\right)$. The dashed and dotted lines are leastsquares fits to the $\beta$ - and $\beta^{*}$-data of PCT and the copolymer having a PET:PCT ratio of 69:31, respectively

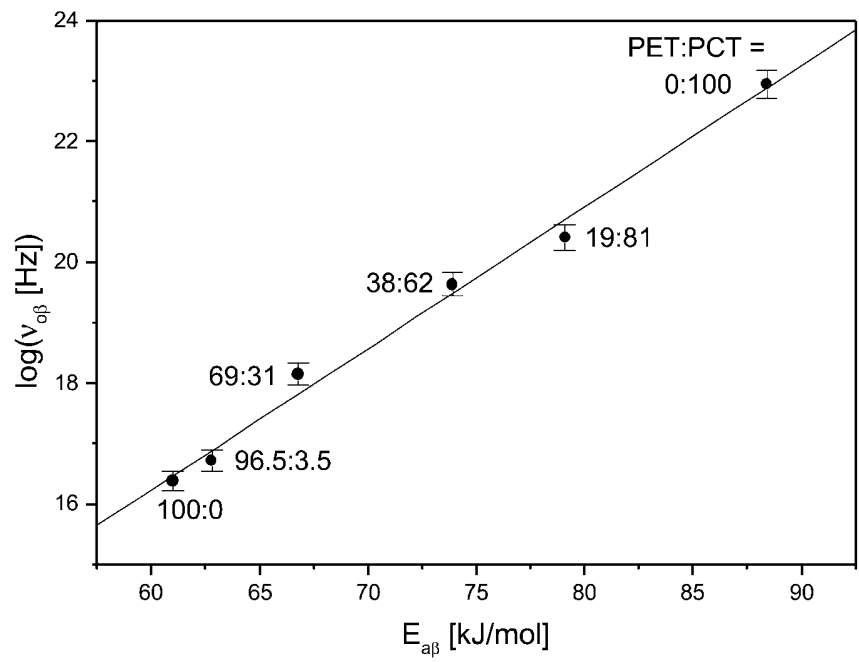

Fig. $10 \log \left(v_{\mathrm{o} \beta}\right)$ versus $E_{\mathrm{a} \beta}$ for the $\beta$-process for various PET-PCT copolymers. The solid line is a linear fit of the compensation law, $\log \left(v_{\mathrm{o} \beta}\right)=2.5+0.2 E_{\mathrm{a}}$
Table 4 Activation energies, $E_{\text {a }}$, and characteristic frequencies, $v_{\mathrm{o}}$, of the $\beta^{*}$ - and $\beta$-relaxations

\begin{tabular}{llllll}
\hline $\begin{array}{l}\text { PET:PCT } \\
\text { ratio }\end{array}$ & \multicolumn{3}{l}{$\beta^{*}$-relaxation } & & $\beta$-relaxation \\
\cline { 2 - 3 } \cline { 5 - 6 } & $E_{\mathrm{a} \pm 0.5(\mathrm{~kJ} / \mathrm{mol})}$ & $\log \left[v_{\mathrm{o}}(\mathrm{Hz})\right] \pm 0.7$ & & $E_{\mathrm{a}} \pm 0.8(\mathrm{~kJ} / \mathrm{mol})$ & $\log \left[v_{\mathrm{o}}(\mathrm{Hz})\right] \pm 0.6$ \\
\hline $100: 0(\mathrm{PET})$ & - & - & 61.0 & 16.4 \\
$96.5: 3.5$ & - & - & 62.8 & 16.7 \\
$69: 31$ & 46.8 & 14.9 & 66.8 & 18.1 \\
$38: 62$ & 46.6 & 12.4 & 73.9 & 19.6 \\
$19: 81$ & 50.2 & 13.0 & 79.1 & 20.4 \\
$0: 100(\mathrm{PCT})$ & 53.1 & 13.6 & 88.4 & \\
\hline
\end{tabular}

cyclohexylene units makes the librational motion of the carbonyl groups more complicated than in pure PET (Fig. 8). In PET-PCT copolymers, such a process seems to be possible only if both the cyclohexylene and carbonyl units move cooperatively together. It seems conceivable that such a cooperative movement should have a higher activation energy than a local one. Because the degree of cooperativity should depend on the number of cyclohexylene units, the activation energy should increase likewise, which we indeed observe (Table 4). This proves that the $\beta$-relaxation is not a purely local relaxation process any longer and indicates an intramolecular coupling to the dynamics of the cyclohexylene ring.

\section{Conclusion}

Broadband dielectric spectroscopy enables the inter- and intramolecular dynamics in a series of random copoly- mers based on PET and PCT to be analyzed. Besides the dynamic glass transition ( $\alpha$-relaxation) and a relaxational process $\left(\alpha^{*}\right.$-relaxation) which is attributed to interfacial zones between the amorphous and the crystalline regions of the copolymer, two intramolecular fluctuations $\left(\beta\right.$ - and $\beta^{*}$-relaxation) are observed: the $\beta$-relaxation corresponding to local fluctuations of the ester groups and an additional relaxation process $\left(\beta^{*}\right)$ which is associated with specific interactions between the ester group and the "chair-chair" conformational transitions of the cyclohexylene ring. The fact that the activation parameters (preexponential factor and activation energy) of the $\beta$-peak are affected by the presence of the CHDM linkages signifies that this process involves intramolecular interactions and that it loses its local nature.

Acknowledgements A stipend of the Deutscher Akademischer Austauschdienst to A.S.M. and financial support by the German Science Foundation within the framework of the Sonderforschungsbereich 294 are greatly acknowledged. 


\section{References}

1. Coburn JC, Boyd RH (1986) Macromolecules 19:2238

2. Ezquerra TA, Balta-Calleja FJ, Zachmann HG (1993) Acta Polym 44:18

3. Ward IM, Maxwell AS, Monnerie L (1998) Polymer 39:6851

4. Gallagher KP, Zhang X, Runt JP (1993) Macromolecules 26:588

5. Runt J, Lei D, Martynowicz LM, Brezny DM, Mayo M (1989) Macromolecules 22:3908

6. Ezquerra TA, Balta-Calleja FJ, Zachmann HG (1994) Polymer 35:1994

7. Reddish W (1950) Trans Faraday Soc 46:459

8. Chen LP, Yee AF, Goetz JM, Schaefer J (1998) Macromolecules 31:5371

9. Ward IM, Maxwell AS, Monnerie L, Laupretre F (1998) Polymer 39:6835

10. Havriliak S, Negami S (1966) J Polym Sci C 14:99
11. Williams G (1979) Adv Polym Sci $13: 59$

12. Williams ML, Landel RF, Ferry JD (1955) J Am Chem Soc 77:3701

13. Blom HP, Teh JW, Bremner T, Rudin A (1998) Polymer 39:4011

14. Fytas G (1989) Macromolecules 22:21

15. Ribes-Greus A, Gomez-Ribelles JL, Diaz-Calleja R (1985) Polymer 26:1849

16. Heijboer J (1977) Int J Polym Mater 6:11

17. Ferry JD (1980) Viscoelastic properties of polymers, 3rd edn. Wiley, New York

18. Baily TT, North AM, Pethrick KA (1981) Molecular motions in high polymers. Clarendon, Oxford

19. McCrum NG, Read BE, Williams G (1967) Anelastic and dielectric effects in polymeric solids. Wiley, London

20. Boye CA (1961) J Polym Sci 55:275
21. Matsuoka S, Quan X (1991) Macromolecules 24:2770

22. Vallerien SU, Kremer F, Boeffel C (1989) Liq Cryst 4:79

23. Dyre JC (1986) J Phys C Solid State Phys 19:5655

24. Yelon A, Movaghar B, Branz HM (1992) Phys Rev B 46:12244

25. Dufresne A, Lavergne C, Lacabanne C (1964) Solid State Commun 29:1094

26. Lacabanne C, Lamure A, Teyssedre G, Bernes A, Mourgues M (1994) NonCryst Solids 172-174:884

27. Hoffman JD, Williams G, Passaglia E (1966) J Polym Sci Part C 14:173

28. Schönhals A, Gessner U, Rübner J (1995) Macromol Chem Phys 196: 1671

29. Schönhals A, Wolf D, Springer J (1995) Macromolecules 28:6254 